\title{
Pairs of a tree and a nontree graph with the same status sequence*
}

\author{
$\mathrm{Pu}$ Qiao, Xingzhi Zhan ${ }^{\dagger}$ \\ Department of Mathematics, East China Normal University, Shanghai 200241, China
}

\begin{abstract}
The status of a vertex $x$ in a graph is the sum of the distances between $x$ and all other vertices. Let $G$ be a connected graph. The status sequence of $G$ is the list of the statuses of all vertices arranged in nondecreasing order. $G$ is called status injective if all the statuses of its vertices are distinct. Let $G$ be a member of a family of graphs $\mathscr{F}$ and let the status sequence of $G$ be $s . G$ is said to be status unique in $\mathscr{F}$ if $G$ is the unique graph in $\mathscr{F}$ whose status sequence is $s$. In 2011, J.L. Shang and C. Lin posed the following two conjectures. Conjecture 1: A tree and a nontree graph cannot have the same status sequence. Conjecture 2: Any status injective tree is status unique in all connected graphs. We settle these two conjectures negatively. For every integer $n \geq 10$, we construct a tree $T_{n}$ and a unicyclic graph $U_{n}$, both of order $n$, with the following two properties: (1) $T_{n}$ and $U_{n}$ have the same status sequence; (2) for $n \geq 15$, if $n$ is congruent to 3 modulo 4 then $T_{n}$ is status injective and among any four consecutive even orders, there is at least one order $n$ such that $T_{n}$ is status injective.
\end{abstract}

Key words. Status; status unique; distance; tree; unicyclic graph

\section{Introduction}

We consider finite simple graphs. The order of a graph is the number of its vertices. A connected graph is said to be unicyclic if it has exactly one cycle. We denote by $V(G)$

\footnotetext{
${ }^{*}$ E-mail addresses: 235711gm@sina.com(P.Qiao), zhan@math.ecnu.edu.cn(X.Zhan).
}

$\dagger$ Corresponding author. 
and $E(G)$ the vertex set and edge set of a graph $G$ respectively. The distance between two vertices $x$ and $y$ in a graph is denoted by $d(x, y)$. The status of a vertex $x$ in a graph $G$, denoted by $s(x)$, is the sum of the distances between $x$ and all other vertices; i.e.,

$$
s(x)=\sum_{y \in V(G)} d(x, y) .
$$

The status sequence of $G$ is the list of the statuses of all vertices of $G$ arranged in nondecreasing order. $G$ is called status injective if all the statuses of its vertices are distinct [2, p.185]. Harary [4] investigated the digraph version of the concept of status in a sociometric framework, while Entringer, Jackson and Snyder [3] studied basic properties of this concept for graphs.

A natural question is: Which graphs are determined by their status sequences? Slater [7] constructed infinitely many pairs of non-isomorphic trees with the same status sequence. Shang [5] gave a method for constructing general non-isomorphic graphs with the same status sequence. Let $G$ be a member of a family of graphs $\mathscr{F}$ and let the status sequence of $G$ be $s . G$ is said to be status unique in $\mathscr{F}$ if $G$ is the unique graph in $\mathscr{F}$ whose status sequence is $s$. Here we view two isomorphic graphs as the same graph. It is known that [6] spiders are status unique in trees and that [1] status injective trees are status unique in trees.

Shang and Lin [6, p.791] posed the following two conjectures in 2011.

Conjecture 1. A tree and a nontree graph cannot have the same status sequence.

Conjecture 2. Any status injective tree is status unique in all connected graphs.

In this paper we settle these two conjectures negatively. For every integer $n \geq 10$, we construct a tree $T_{n}$ and a unicyclic graph $U_{n}$, both of order $n$, with the same status sequence. There are infinitely many odd orders $n$ and infinitely many even orders $n$ such that $T_{n}$ is status injective.

\section{Main Results}

We will need the following lemmas. For a set $S$, the notation $|S|$ denotes the cardinality of $S$.

Lemma 1. [3, p.284] Let $x y$ be an edge of a tree $T$ and let $T_{1}$ and $T_{2}$ be the two components of $T-x y$ with $x \in V\left(T_{1}\right)$ and $y \in V\left(T_{2}\right)$. Then $s(y)=s(x)+\left|V\left(T_{1}\right)\right|-\left|V\left(T_{2}\right)\right|$. 
Lemma 2. Let $x_{0} x_{1} x_{2} \ldots x_{k}$ be a path in a tree and denote $d=s\left(x_{1}\right)-s\left(x_{0}\right)$. Then $s\left(x_{j+1}\right)-s\left(x_{j}\right) \geq d+2 j$ for each $j=1,2, \ldots, k-1$. Consequently if $s\left(x_{0}\right) \leq s\left(x_{1}\right)$ then $s\left(x_{j+1}\right)-s\left(x_{j}\right) \geq 2 j$ for each $j=1,2, \ldots, k-1$ and in particular, $s\left(x_{1}\right)<s\left(x_{2}\right)<s\left(x_{3}\right)<$ $\cdots<s\left(x_{k}\right)$.

Proof. It suffices to prove the first assertion. We first show the following

Claim. If $x y z$ is a path in a tree and denote $c=s(y)-s(x)$, then $s(z)-s(y) \geq c+2$.

Let $T$ be the tree of order $n$. Let $A$ and $B$ be the two components of $T-x y$ with $x \in V(A)$ and $y \in V(B)$, and let $G$ and $H$ be the two components of $T-y z$ with $y \in V(G)$ and $z \in V(H)$. By Lemma 1, s(y) -s(x)=|V(A)|-|V(B)|=c. We also have $|V(A)|+|V(B)|=n$ since every edge in a tree is a cut-edge. Hence $2|V(A)|=c+n$. Since $V(A) \subset V(G)$ and $y \in V(G)$ but $y \notin V(A)$, we have $|V(G)| \geq|V(A)|+1$. By Lemma 1 and the relation $|V(G)|+|V(H)|=n$ we deduce

$$
s(z)-s(y)=|V(G)|-|V(H)|=2|V(G)|-n \geq 2|V(A)|+2-n=c+2 .
$$

This proves the claim.

Applying the claim successively to the path $x_{i-1} x_{i} x_{i+1}$ for $i=1,2, \ldots, k-1$ we obtain the first assertion in Lemma $2 . \square$

Lemma 2 is a generalization and strengthening of a result in [3, p.291], which states that if $x_{0} x_{1} \ldots x_{k}$ is a path in a tree and $x_{0}$ has the minimum status of all vertices, then $s\left(x_{1}\right)<s\left(x_{2}\right)<\cdots<s\left(x_{k}\right)$.

Lemma 3. The quadratic polynomial equation

$$
p^{2}+5 p+4=q^{2}+q-6
$$

in $p$ and $q$ has no nonnegative integer solution.

Proof. Suppose that $p$ and $q$ are nonnegative integers. If $q \leq p+2$, then $q^{2}+q-6 \leq$ $(p+2)^{2}+(p+2)-6=p^{2}+5 p<p^{2}+5 p+4$. If $q \geq p+3$, then $q^{2}+q-6 \geq$ $(p+3)^{2}+(p+3)-6=p^{2}+7 p+6>p^{2}+5 p+4$. Hence the equation cannot have any nonnegative integer solution.

Remark. It is not hard to prove that the only integer solutions of the equation in Lemma 3 are $(p, q)=(-4,-3),(-4,2),(-1,-3),(-1,2)$.

Denote by $\mathbb{N}$ the set of positive integers. 
Lemma 4. Let the two functions $f(p)=p^{2}+5 p+4$ and $h(q)=q^{2}+q-6$ be defined on the set $\mathbb{N}$. If $p \geq 7$ and $|f(p)-h(q)| \leq 15$, then $q=p+2$ and $f(p)-h(q)=4$.

Proof. If $q \geq p+3$, then

$$
h(q) \geq h(p+3)=f(p)+2 p+2 \geq f(p)+16 .
$$

If $p-2 \leq q \leq p+1$, then

$$
f(p) \geq f(q-1)=h(q)+2 q+6 \geq h(q)+2 p+2 \geq h(q)+16 .
$$

If $q \leq p-3$, then

$$
f(p) \geq f(q+3)=h(q)+10 q+34 \geq h(q)+44 .
$$

Hence we must have $q=p+2$ and in this case, $f(p)-h(q)=4$.

Now we are ready to state and prove the main result.

Theorem 5. For every integer $n \geq 10$, there exists a tree $T_{n}$ and a unicyclic graph $U_{n}$, both of order $n$, with the following two properties:

(1) $T_{n}$ and $U_{n}$ have the same status sequence;

(2) for $n \geq 15$, if $n \equiv 3(\bmod 4)$ then $T_{n}$ is status injective and among any four consecutive even orders, there is at least one order $n$ such that $T_{n}$ is status injective.

Proof. For the orders $n \geq 19$ we have a uniform construction of $T_{n}$ and $U_{n}$, and we treat this case first. For the orders $10 \leq n \leq 18$, the graphs will be constructed individually and they appear at the end of this proof.

Now suppose $n \geq 19$. We distinguish the odd orders and the even orders. Let $n=2 k+5$ with $k \geq 7$. We define $T_{n}$ and $U_{n}$ as follows. $V\left(T_{n}\right)=\left\{x_{i} \mid i=1,2, \ldots, 2 k+5\right\}$ and $E\left(T_{n}\right)=$

$$
\left\{x_{i} x_{i+1} \mid i=1,2, \ldots, 2 k-1\right\} \cup\left\{x_{3} x_{2 k+1}, x_{k+1} x_{2 k+5}, x_{k+3} x_{2 k+4}, x_{2 k-3} x_{2 k+3}, x_{2 k-2} x_{2 k+2}\right\}
$$

$V\left(U_{n}\right)=\left\{y_{i} \mid i=1,2, \ldots, 2 k+5\right\}$ and $E\left(U_{n}\right)=\left\{y_{i} y_{i+1} \mid i=1,2, \ldots, 2 k-1\right\} \cup\left\{y_{5} y_{2 k+3}, y_{k-1} y_{2 k+4}\right.$, $\left.y_{k+1} y_{2 k+5}, y_{2 k-2} y_{2 k+2}, y_{2 k-1} y_{2 k+1}, y_{2 k+1} y_{2 k+2}\right\}$. Note that $T_{n}$ is a caterpillar of maximum degree 3 and $U_{n}$ is a unicyclic graph. $T_{n}$ and $U_{n}$ are illustrated in Figure 1. 

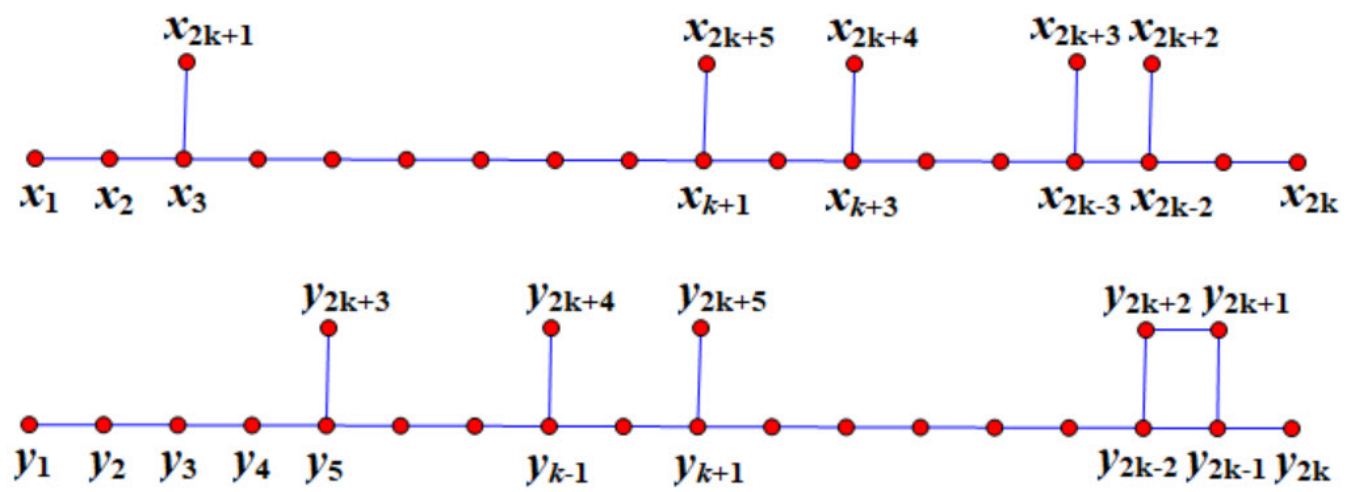

FIGURE $1 \quad \mathrm{~T}_{\mathrm{n}}$ and $\mathrm{U}_{\mathrm{n}}$ with $\mathrm{n}=2 \mathrm{k}+5$ and $\mathrm{k} \geq 7$

It can be checked directly that $s\left(x_{i}\right)=s\left(y_{i}\right)$ for $i=1,2,3, k+1,2 k-1,2 k, \ldots, 2 k+5$ and $s\left(x_{i}\right)=s\left(y_{2 k+2-i}\right)$ for $4 \leq i \leq 2 k-2$. Hence, $T_{n}$ and $U_{n}$ have the same status sequence. For the even orders $n=2 k+6$ with $k \geq 7, T_{n}$ is obtained from $T_{n-1}$ defined above by adding the edge $x_{2 k+5} x_{2 k+6}$, and $U_{n}$ is obtained from $U_{n-1}$ defined above by adding the edge $y_{2 k+5} y_{2 k+6} . T_{n}$ and $U_{n}$ are illustrated in Figure 2 .

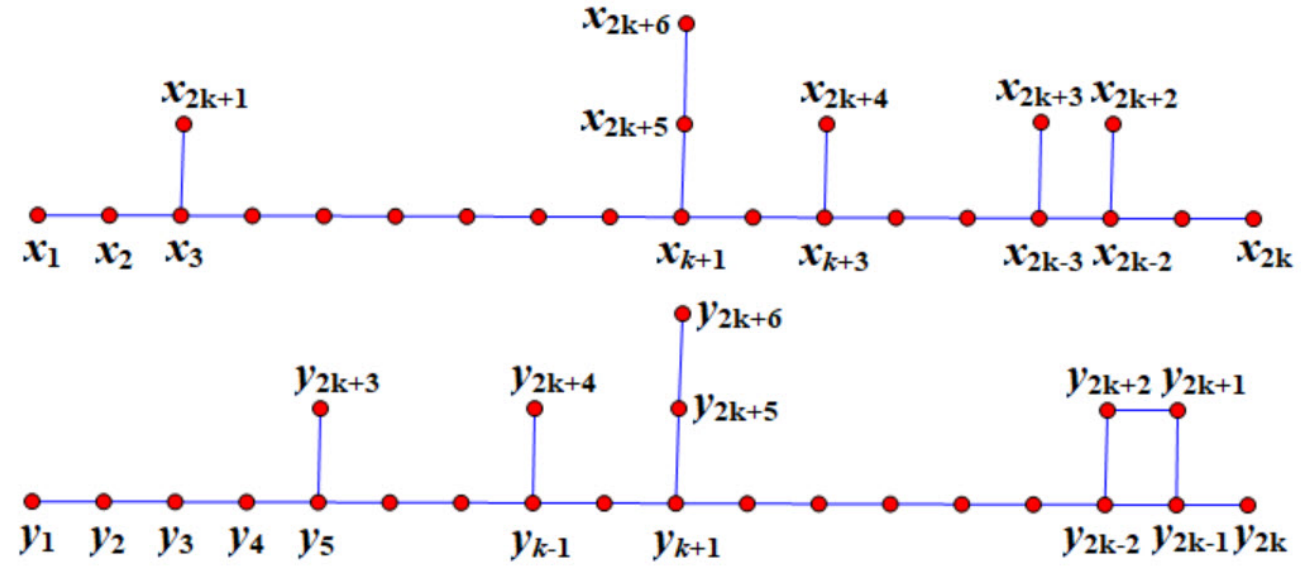

FIGURE $2 T_{n}$ and $U_{n}$ with $n=2 k+6$ and $\mathrm{k} \geq 7$

We check easily that $s\left(x_{i}\right)=s\left(y_{i}\right)$ for $i=1,2,3, k+1,2 k-1,2 k, \ldots, 2 k+6$ and $s\left(x_{i}\right)=s\left(y_{2 k+2-i}\right)$ for $4 \leq i \leq 2 k-2$. Thus $T_{n}$ and $U_{n}$ also have the same status sequence.

Next we prove that the trees $T_{n}$ satisfy condition (2) in Theorem 5 . In fact, we will determine precisely for which orders $n, T_{n}$ is status injective.

First consider the case when $n$ is odd and let $n=2 k+5$ with $k \geq 7$. Denote $a=$ 
$s\left(x_{k+1}\right)=k^{2}+3 k-2$. We have

$$
\begin{aligned}
& s\left(x_{k-p}\right)= \begin{cases}a+(p+2)^{2}-1 & \text { if } \quad 0 \leq p \leq k-3, \\
a+k^{2}+1 & \text { if } p=k-2, \\
a+(k+1)^{2}+3 & \text { if } p=k-1 ;\end{cases} \\
& s\left(x_{k+q}\right)= \begin{cases}a+1 & \text { if } q=2, \\
a+q^{2}-5 & \text { if } 3 \leq q \leq k-3, \\
a+(q+2)^{2}-4 k+1 & \text { if } k-2 \leq q \leq k ;\end{cases} \\
& s\left(x_{2 k+r}\right)= \begin{cases}a+(k+1-r)^{2}+3 & \text { if } 1 \leq r \leq 3, \\
a+2 k+7 & \text { if } r=4, \\
a+2 k+3 & \text { if } r=5 .\end{cases}
\end{aligned}
$$

In calculating the values $s\left(x_{i}\right)$ for $1 \leq i \leq 2 k$ we have used the fact that if $P=z_{1} z_{2} \ldots z_{m}$ is a path, then

$$
s\left(z_{i}\right)=i(i-m-1)+m(m+1) / 2
$$

in $P$, while in calculating the values $s\left(x_{j}\right)$ for $j=2 k+1, \ldots, 2 k+5$ we have used Lemma 1. From the above expressions it follows that $x_{k+1}$ is the unique vertex with the minimum status, $x_{1}, x_{2}, x_{3}, x_{2 k-1}, x_{2 k}, x_{2 k+1}, x_{2 k+2}, x_{2 k+3}$ are the vertices with the eight largest statuses, since

$$
s\left(x_{1}\right)>s\left(x_{2 k}\right)>s\left(x_{2 k+1}\right)>s\left(x_{2}\right)>s\left(x_{2 k+2}\right)>s\left(x_{2 k-1}\right)>s\left(x_{3}\right)>s\left(x_{2 k+3}\right)>s\left(x_{i}\right)
$$

for any $i \neq 1,2,3,2 k-1,2 k, 2 k+1,2 k+2,2 k+3$ and

$$
s\left(x_{2 k+1}\right)>s\left(x_{2 k+2}\right)>s\left(x_{2 k+3}\right)>s\left(x_{2 k+4}\right)>s\left(x_{2 k+5}\right) .
$$

Partition the vertex set of $T_{n}$ into three sets:

$$
L=\left\{x_{i} \mid 1 \leq i \leq k\right\}, \quad R=\left\{x_{i} \mid k+1 \leq i \leq 2 k\right\} \text { and } W=\left\{x_{i} \mid 2 k+1 \leq i \leq 2 k+5\right\} .
$$

The inequalities in (2) show that any two distinct vertices in $W$ have different statuses. Applying Lemma 2 to the two paths $x_{k+1} x_{k} x_{k-1} \ldots x_{2} x_{1}$ and $x_{k+1} x_{k+2} \ldots x_{2 k-1} x_{2 k}$ we see that any two distinct vertices in $L$ or in $R$ have different statuses. Next we show that for any $x \in L$ and $y \in R, s(x) \neq s(y)$. By the inequalities in (1) it suffices to prove that $s\left(x_{i}\right) \neq s\left(x_{j}\right)$ for $4 \leq i \leq k$ and $k+2 \leq j \leq 2 k-2$, which is equivalent to $s\left(x_{k-p}\right) \neq s\left(x_{k+q}\right)$ 
for $0 \leq p \leq k-4$ and $2 \leq q \leq k-2$. We have the expressions $s\left(x_{k-p}\right)=a+(p+2)^{2}-1$ for $0 \leq p \leq k-4, s\left(x_{k+2}\right)=a+1, s\left(x_{2 k-2}\right)=a+k^{2}-4 k+1$ and $s\left(x_{k+q}\right)=a+q^{2}-5$ for $3 \leq q \leq k-3$. First, $s\left(x_{k-p}\right) \geq s\left(x_{k}\right)=a+3>a+1=s\left(x_{k+2}\right)$. The equality $s\left(x_{k-p}\right)=s\left(x_{k+q}\right)$ for $3 \leq q \leq k-3$ is equivalent to $4=(q+p+2)(q-p-2)$, which is impossible, since $q+p+2 \geq 5$ and $q-p-2$ is an integer. Also, $s\left(x_{k-p}\right)=s\left(x_{2 k-2}\right)$ is equivalent to $2=(k+p)(k-p-4)$, which is impossible, since $k+p \geq 7$ and $k-p-4$ is an integer. Hence $s(x) \neq s(y)$ for $x \in L$ and $y \in R$.

By the above analysis, it is clear that the only possibilities for two distinct vertices to have the same status are $s\left(x_{2 k+5}\right)=s\left(x_{i}\right)$ and $s\left(x_{2 k+4}\right)=s\left(x_{i}\right)$ for $4 \leq i \leq k$ or $k+2 \leq i \leq 2 k-2$. By the expressions for their status values, it is easy to verify that $s\left(x_{2 k+5}\right)=s\left(x_{i}\right)$ for some $i$ with $4 \leq i \leq k$ if and only if $k=2 c^{2}-2$ for some integer $c$; $s\left(x_{k+2}\right)<s\left(x_{2 k+5}\right)<s\left(x_{2 k-2}\right)$ and $s\left(x_{2 k+5}\right)=s\left(x_{i}\right)$ for some $i$ with $k+3 \leq i \leq 2 k-3$ if and only if $k=2 c^{2}-4$ for some integer $c ; s\left(x_{2 k+4}\right)=s\left(x_{i}\right)$ for some $i$ with $4 \leq i \leq k$ if and only if $k=2 c^{2}-4$ for some integer $c ; s\left(x_{k+2}\right)<s\left(x_{2 k+4}\right)<s\left(x_{2 k-2}\right)$ and $s\left(x_{2 k+4}\right)=s\left(x_{i}\right)$ for some $i$ with $k+3 \leq i \leq 2 k-3$ if and only if $k=2 c^{2}-6$ for some integer $c$.

Thus, $T_{n}$ with $n=2 k+5$ is not status injective if and only if $k=2 c^{2}-2,2 c^{2}-4$ or $2 c^{2}-6$ for some integer $c$. Since all these values of $k$ are even, it follows that for every odd $k, T_{n}$ is status injective; i.e., if $n \equiv 3(\bmod 4)$ then $T_{n}$ is status injective.

Next we treat the case when the order $n$ is even. Let $n=2 k+6$ with $k \geq 7$. With $d=s\left(x_{k+1}\right)=k^{2}+3 k$ we have

$$
\begin{gathered}
s\left(x_{k-p}\right)= \begin{cases}d+p^{2}+5 p+4 & \text { if } 0 \leq p \leq k-3, \\
d+k^{2}+k & \text { if } p=k-2, \\
d+k^{2}+3 k+4 & \text { if } p=k-1 ;\end{cases} \\
s\left(x_{k+q}\right)= \begin{cases}d+2 & \text { if } q=2, \\
d+q^{2}+q-6 & \text { if } 3 \leq q \leq k-3, \\
d+q^{2}+5 q-4 k+4 & \text { if } k-2 \leq q \leq k ;\end{cases}
\end{gathered}
$$




$$
s\left(x_{2 k+r}\right)= \begin{cases}d+k^{2}+k+2 & \text { if } r=1, \\ d+k^{2}-k+2 & \text { if } r=2, \\ d+k^{2}-3 k+4 & \text { if } r=3, \\ d+2 k+10 & \text { if } r=4, \\ d+2 k+2 & \text { if } r=5, \\ d+4 k+6 & \text { if } r=6 .\end{cases}
$$

From the above expressions we deduce that $x_{k+1}$ is the unique vertex with the minimum status $d$. The case $k=7$ corresponds to $n=20$ and we check directly that $T_{20}$ is status injective. Next suppose $k \geq 8$. Then $x_{1}, x_{2}, x_{3}, x_{2 k-1}, x_{2 k}, x_{2 k+1}, x_{2 k+2}, x_{2 k+3}$ are the vertices with the eight largest statuses, since

$$
s\left(x_{1}\right)>s\left(x_{2 k}\right)>s\left(x_{2 k+1}\right)>s\left(x_{2}\right)>s\left(x_{2 k+2}\right)>s\left(x_{2 k-1}\right)>s\left(x_{3}\right)>s\left(x_{2 k+3}\right)>s\left(x_{i}\right)
$$

for any $i \neq 1,2,3,2 k-1,2 k, 2 k+1,2 k+2,2 k+3$. Also

$$
s\left(x_{2 k+1}\right)>s\left(x_{2 k+2}\right)>s\left(x_{2 k+3}\right)>s\left(x_{2 k+6}\right)>s\left(x_{2 k+4}\right)>s\left(x_{2 k+5}\right) .
$$

In considering two vertices with equal status, we can exclude the eight vertices with the eight largest statuses by (3) and the unique vertex $x_{k+1}$ with the minimum status. Denote $L^{\prime}=\left\{x_{i} \mid 4 \leq i \leq k\right\}, \quad R^{\prime}=\left\{x_{i} \mid k+2 \leq i \leq 2 k-2\right\}$ and $W^{\prime}=\left\{x_{i} \mid 2 k+1 \leq i \leq 2 k+6\right\}$.

Let $x$ and $y$ be two distinct vertices with $s(x)=s(y)$. By the inequalities in (4), it is impossible that $x, y \in W^{\prime}$. By Lemma 2 we cannot have $x, y \in L^{\prime}$ or $x, y \in R^{\prime}$. Suppose $x \in L^{\prime}$ and $y \in R^{\prime}$. We have $s(x)>s\left(x_{k+2}\right), s\left(x_{4}\right)>s\left(x_{2 k-2}\right)$ and $s\left(x_{i}\right)<s\left(x_{2 k-2}\right)$ for $5 \leq i \leq k$. Thus, $y \neq x_{k+2}, x_{2 k-2}$. We have $x=x_{i}$ for some $i$ with $4 \leq i \leq k$ and $y=x_{j}$ for some $j$ with $k+3 \leq j \leq 2 k-3$. Hence $s(x)=d+p^{2}+5 p+4$ with $0 \leq p \leq k-4$ and $s(y)=d+q^{2}+q-6$ with $3 \leq q \leq k-3$. Then $s(x)=s(y)$ yields $p^{2}+5 p+4=q^{2}+q-6$, which is impossible by Lemma 3 .

Now, by (3) and the above analysis it is clear that $s(x)=s(y)$ can occur only if $x \in\left\{x_{2 k+4}, x_{2 k+5}, x_{2 k+6}\right\}$ and $y \in L^{\prime} \cup R^{\prime}$ or the roles of $x$ and $y$ are interchanged. The case $k=8$ corresponds to $n=22$, and we check directly that $T_{22}$ is not status injective. Next we suppose $k \geq 9$. Then $s\left(x_{2 k-2}\right)>s\left(x_{2 k+6}\right)>s\left(x_{2 k+4}\right)>s\left(x_{2 k+5}\right)$, and hence $x_{2 k-2}$ can be excluded from $R^{\prime}$. Similarly, since $s\left(x_{k+2}\right)<s\left(x_{k}\right)<s\left(x_{2 k+5}\right)<s\left(x_{2 k+4}\right)<s\left(x_{2 k+6}\right)$, $x_{k}$ can be excluded from $L^{\prime}$ and $x_{k+2}$ can be excluded from $R^{\prime}$. Note that the statuses of the vertices in $L^{\prime} \backslash\left\{x_{k}\right\}$ have the uniform expression $d+p^{2}+5 p+4$ with $1 \leq p \leq k-4$ and 
the statuses of the vertices in $R^{\prime} \backslash\left\{x_{k+2}, x_{2 k-2}\right\}$ have the uniform expression $d+q^{2}+q-6$ with $3 \leq q \leq k-3$.

Denote the empty set by $\phi$, and denote $\Omega_{k}=\{2 k+2,2 k+10,4 k+6\}, \Gamma_{k}=A_{k} \cup B_{k}$ where $A_{k}=\left\{p^{2}+5 p+4 \mid 1 \leq p \leq k-4, p \in \mathbb{N}\right\}$ and $B_{k}=\left\{q^{2}+q-6 \mid 3 \leq q \leq k-3, q \in \mathbb{N}\right\}$. It follows that when $k \geq 9, T_{n}$ has two distinct vertices with the same status if and only if $\Omega_{k} \cap \Gamma_{k} \neq \phi$. Denote $\Gamma=A \cup B$ where $A=\left\{p^{2}+5 p+4 \mid p \in \mathbb{N}\right\}$ and $B=\left\{q^{2}+q-6 \mid q \in \mathbb{N}\right\}$. Since $\Omega_{k} \cap \Gamma_{k}=\Omega_{k} \cap \Gamma$, we obtain the following criterion for $k \geq 9$ :

$T_{n}$ is status injective if and only if $\Omega_{k} \cap \Gamma=\phi$.

The graphs $T_{n}$ with $15 \leq n \leq 18$ constructed below are all status injective. Using the above criterion we can check that $T_{n}$ is status injective for

$$
k=10,14,18,21,23,25,27,29,33,35,38,40,42 .
$$

Thus the assertion in Theorem 5 on $T_{n}$ for even $n$ with $k \leq 42$ is true.

Next we suppose $k \geq 43$. We will prove that among the four numbers $k, k+1, k+2, k+3$ there is at least one for which $T_{n}$ is status injective. To do so, consider

$$
\begin{aligned}
\Omega_{k} & =\{2 k+2,2 k+10,4 k+6\} \\
\Omega_{k+1} & =\{2 k+4,2 k+12,4 k+10\} \\
\Omega_{k+2} & =\{2 k+6,2 k+14,4 k+14\} \\
\Omega_{k+3} & =\{2 k+8,2 k+16,4 k+18\} .
\end{aligned}
$$

The numbers in these four sets can be partitioned into two classes:

$$
X=\{2 k+i \mid i=2,4,6,8,10,12,14,16\} \text { and } Y=\{4 k+j \mid j=6,10,14,18\} .
$$

We claim that

$$
|X \cap A| \leq 1, \quad|X \cap B| \leq 1, \quad|Y \cap A| \leq 1, \quad|Y \cap B| \leq 1
$$

Define two polynomials $f(p)=p^{2}+5 p+4$ and $h(q)=q^{2}+q-6$. Then $A=\{f(p) \mid p \in \mathbb{N}\}$ and $B=\{h(q) \mid q \in \mathbb{N}\}$. In the sequel the symbol $\Rightarrow$ means "implies". We first prove $|X \cap A| \leq 1$. To the contrary, suppose there exist $i, j, p_{1}, p_{2}$ with $2 \leq i<j \leq 16$ and $p_{1}<p_{2}$ such that $f\left(p_{1}\right)=2 k+i$ and $f\left(p_{2}\right)=2 k+j . k \geq 43$ and $i \geq 2 \Rightarrow$ $f\left(p_{1}\right)=2 k+i \geq 88 \Rightarrow p_{1} \geq 7$. We have $f\left(p_{2}\right)-f\left(p_{1}\right)=j-i \leq 14$. But on the other hand, $f\left(p_{2}\right)-f\left(p_{1}\right) \geq f\left(p_{1}+1\right)-f\left(p_{1}\right)=2 p_{1}+6 \geq 20$, a contradiction. The inequality 
$|X \cap B| \leq 1$ is similarly proved by using the fact that $h(q) \in X \Rightarrow h(q) \geq 88 \Rightarrow q \geq 10$. The inequalities $|Y \cap A| \leq 1$ and $|Y \cap B| \leq 1$ can also be similarly proved by using the facts that $f(p) \in Y \Rightarrow f(p) \geq 178 \Rightarrow p \geq 11$ and $h(q) \in Y \Rightarrow h(q) \geq 178 \Rightarrow q \geq 14$.

Note that the assumption $k \geq 43$ implies that $\min X \geq 88$ and $\min Y \geq 178$. Hence if $f(p) \in X \cup Y$ we have $p \geq 7$ and Lemma 4 can be applied.

Suppose $\Omega_{i} \cap \Gamma \neq \phi$ for $i=k, k+1, k+2$. We will show that $\Omega_{k+3} \cap \Gamma=\phi$. Since $\Omega_{k} \cap \Gamma \neq \phi$, at least one of the two cases $\{2 k+2,2 k+10\} \cap \Gamma \neq \phi$ and $4 k+6 \in \Gamma$ must occur. Recall that $\Gamma=A \cup B$.

Case 1. $\{2 k+2,2 k+10\} \cap \Gamma \neq \phi$. We first consider the case when $\{2 k+2,2 k+10\} \cap A \neq$ $\phi$. Denote $\Psi=\{2 k+4,2 k+12,2 k+8,2 k+16\}$. By (5), $\Psi \cap A=\phi$. By Lemma 4 , $\Psi \cap B=\phi$. It follows that $\Psi \cap \Gamma=\phi$. Since $\Omega_{k+1} \cap \Gamma \neq \phi$ and $\{2 k+4,2 k+12\} \cap \Gamma=\phi$, we deduce that $4 k+10 \in \Gamma$. By (5), $4 k+10$ and $4 k+18$ can not be both in $A$ or both in $B$. Since $4 \neq 8=(4 k+18)-(4 k+10) \leq 15$, by Lemma 4 it is also impossible that one of $4 k+10$ and $4 k+18$ is in $A$ and the other in $B$. But $4 k+10 \in \Gamma=A \cup B$. Hence $4 k+18 \notin \Gamma$ and we obtain $\Omega_{k+3} \cap \Gamma=\phi$. The case when $\{2 k+2,2 k+10\} \cap B \neq \phi$ is similar. Again we use (5), Lemma 4 and $\Omega_{k+1} \cap \Gamma \neq \phi$ to deduce $\Omega_{k+3} \cap \Gamma=\phi$.

Case 2. $4 k+6 \in \Gamma$. Using (5) and Lemma 4 we deduce that $\{4 k+14,4 k+18\} \cap \Gamma=\phi$. Then the condition $\Omega_{k+2} \cap \Gamma \neq \phi$ implies $\{2 k+6,2 k+14\} \cap \Gamma \neq \phi$. Applying (5) and Lemma 4 once more we have $\{2 k+8,2 k+16\} \cap \Gamma=\phi$. Hence $\Omega_{k+3} \cap \Gamma=\phi$.

This completes the proof of the case $n \geq 19$ of Theorem 5 . The graph pairs $T_{n}$ and $U_{n}$ with $10 \leq n \leq 18$ are depicted in Figures 3-11 below. They satisfy the condition $s\left(T_{n}\right)=s\left(U_{n}\right)$ and for $15 \leq n \leq 18, T_{n}$ is status injective. In these graphs, the number beside a vertex is the status of that vertex. 

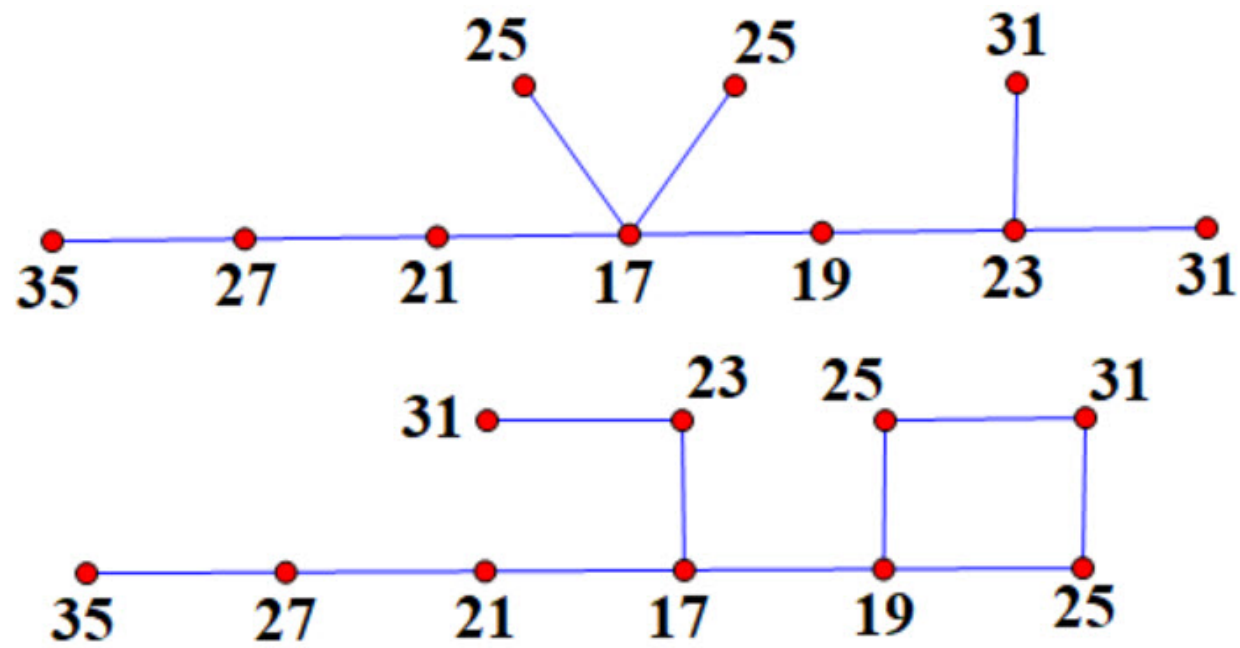

FIGURE $3 \quad \mathrm{~T}_{10}$ and $\mathrm{U}_{10}$
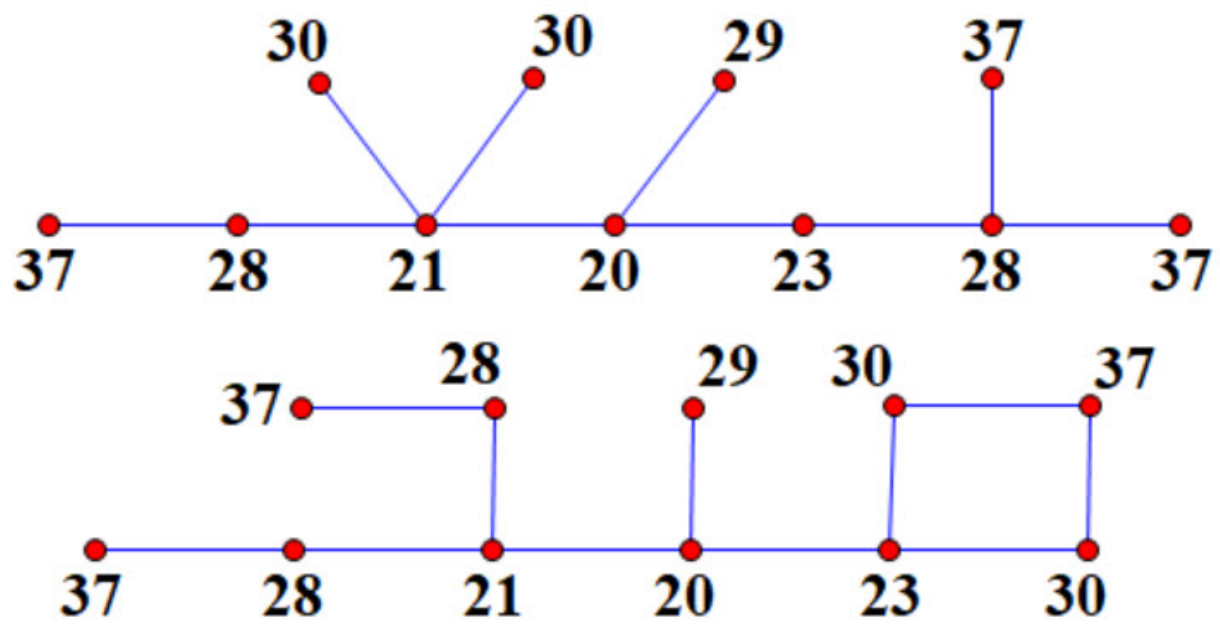

FIGURE $4 \quad T_{11}$ and $U_{11}$
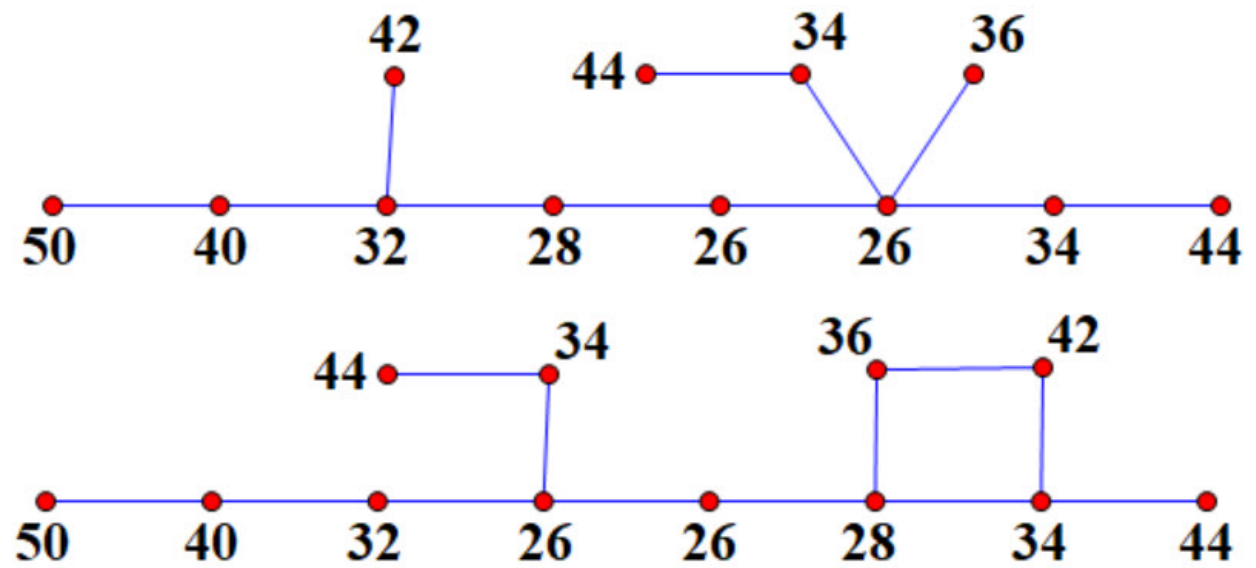

FIGURE $5 \quad \mathrm{~T}_{12}$ and $\mathrm{U}_{12}$ 

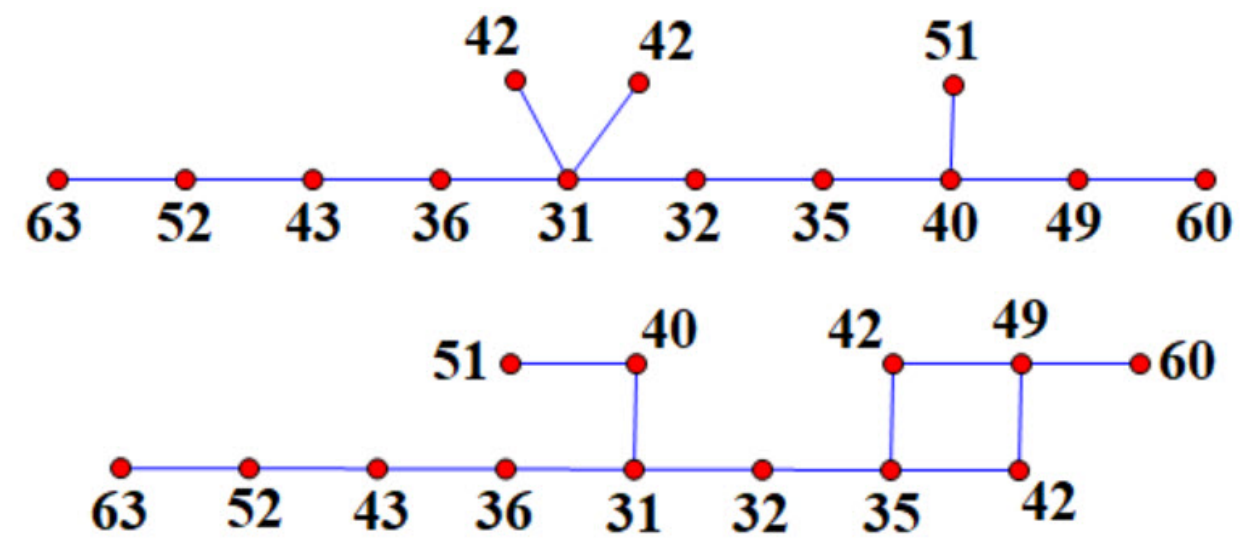

FIGURE $6 \mathrm{~T}_{13}$ and $\mathrm{U}_{13}$
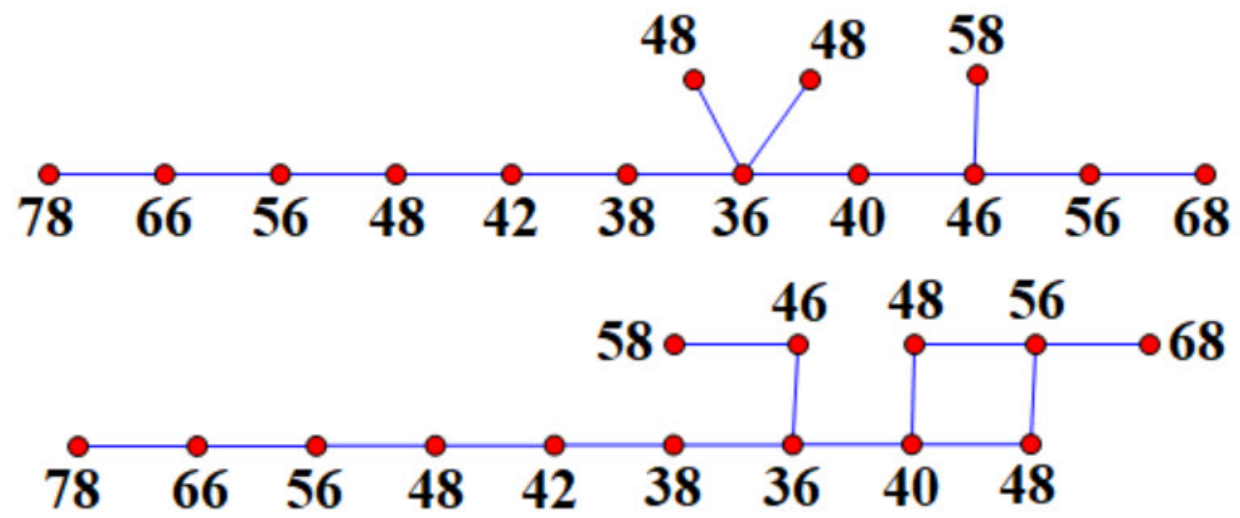

FIGURE $7 \quad \mathrm{~T}_{14}$ and $\mathrm{U}_{14}$
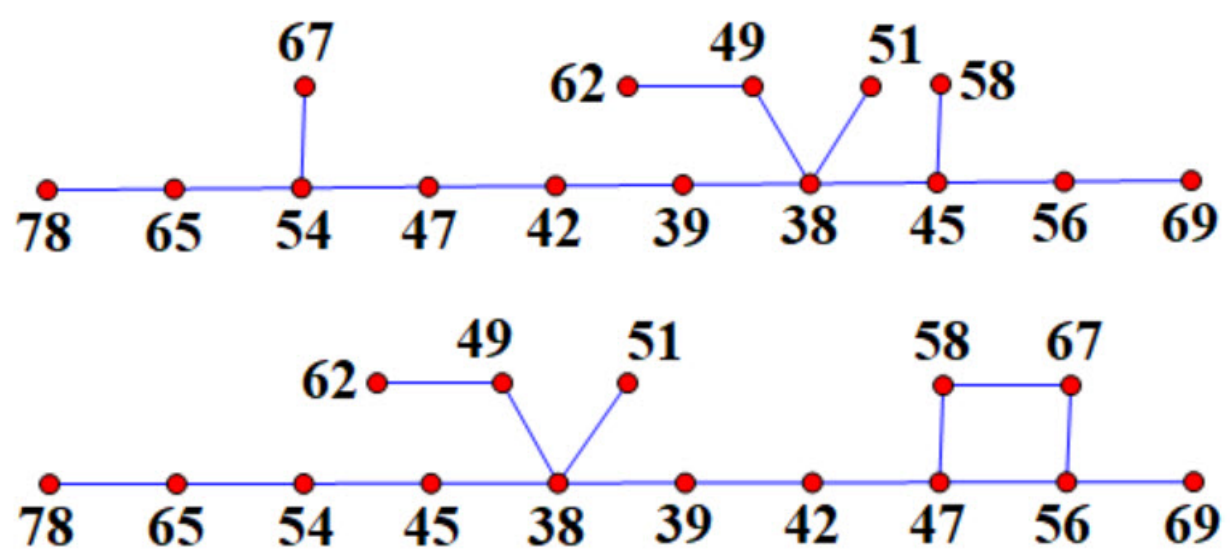

FIGURE $8 \quad \mathrm{~T}_{15}$ and $\mathrm{U}_{15}$ 

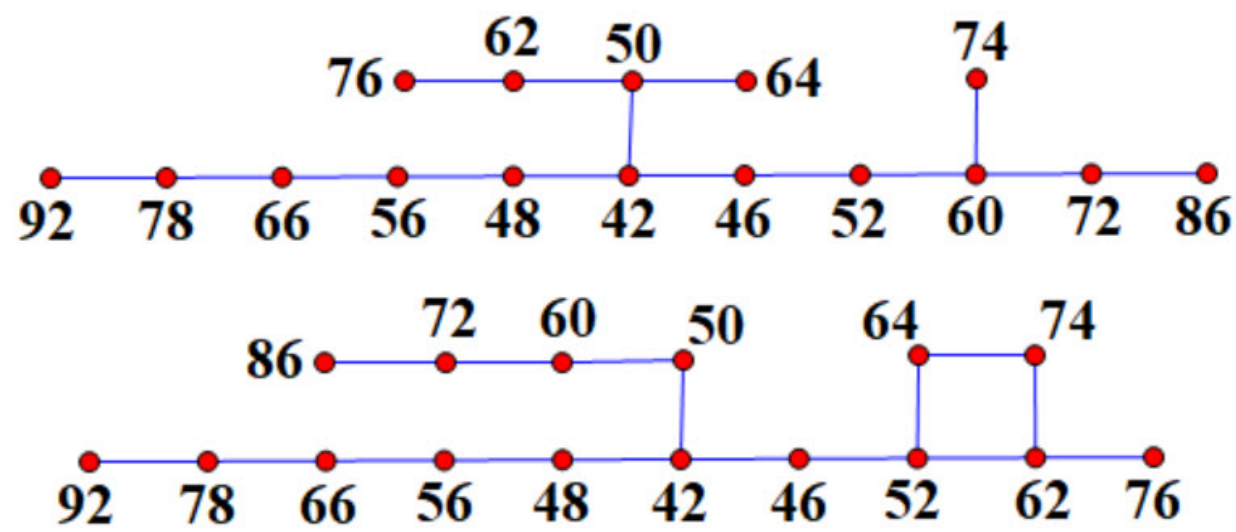

FIGURE $9 \mathrm{~T}_{16}$ and $\mathrm{U}_{16}$
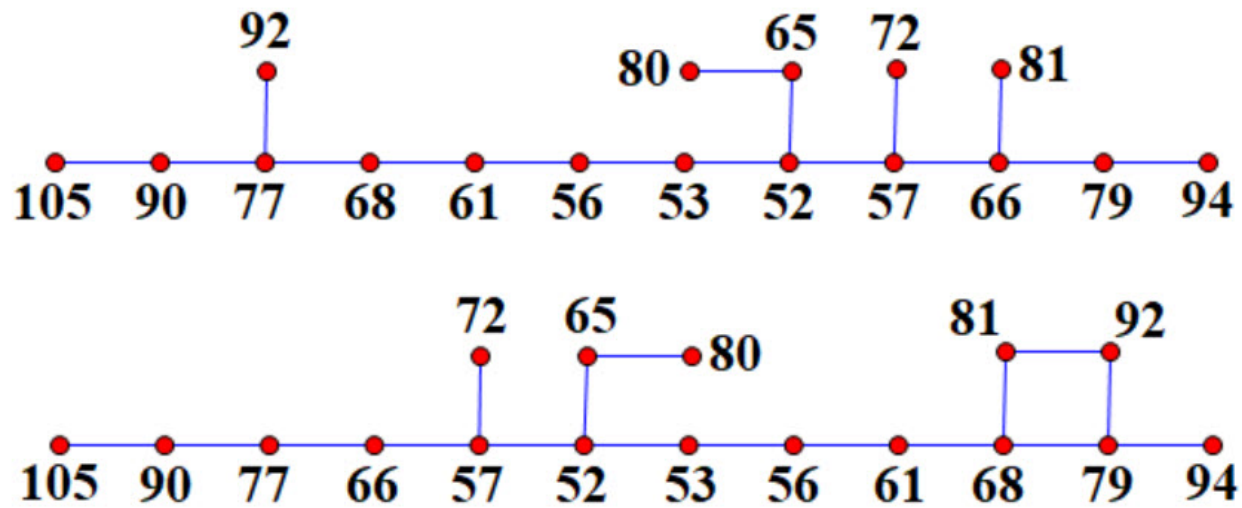

FIGURE $10 \quad \mathrm{~T}_{17}$ and $\mathrm{U}_{17}$

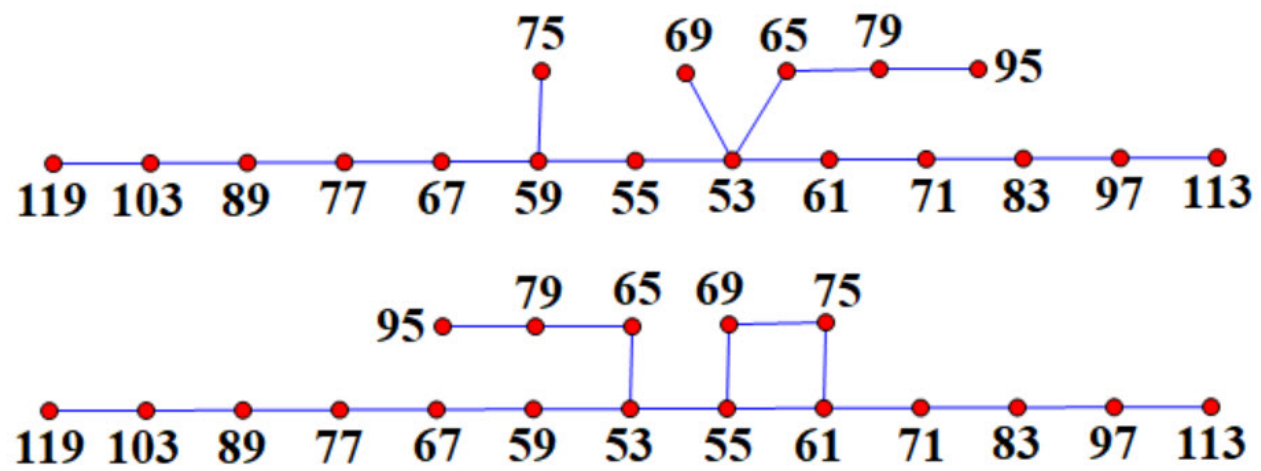

FIGURE $11 \mathrm{~T}_{18}$ and $\mathrm{U}_{18}$

This completes the proof of Theorem $5 . \square$

Remark. A computer search shows that 10 is the smallest order for the existence of a tree and a nontree graph with the same status sequence. 
Acknowledgement. This research was supported by the NSFC grants 11671148 and 11771148 and Science and Technology Commission of Shanghai Municipality (STCSM) grant $18 \mathrm{dz} 2271000$.

\section{References}

[1] A. Abiad, B. Brimkov, A. Chan and A. Grigoriev, On the status sequences of trees, arXiv:1812.03765v1.

[2] F. Buckley and F. Harary, Distance in Graphs, Addison-Wesley Publishing Company, 1990.

[3] R.C. Entringer, D.E. Jackson and D.A. Snyder, Distance in graphs, Czechoslovak Math. J. 26(1976), no.2, 283-296.

[4] F. Harary, Status and contrastatus, Sociometry 22(1959), 23-43.

[5] J.L. Shang, On constructing graphs with the same status sequence, Ars Combin. 113(2014), 429-433.

[6] J.L. Shang and C. Lin, Spiders are status unique in trees, Discrete Math. 311(2011), 785-791.

[7] P.J. Slater, Counterexamples to Randić's conjecture on distance degree sequences for trees, J. Graph Theory 6(1982), 89-92. 